\title{
Granulomatös-nekrotisierende Pneumonie durch Schimmelpilzinfektion bei einer Ziege
}

\author{
Wapf, P ; Ossent, Pete ; Scharf, G ; Braun, Ueli
}

\begin{abstract}
This case report describes the clinical and postmortem findings in a 2.5-year-old goat with necrotizing granulomatous pneumonia. The goat was referred to our clinic because of swelling of the head and neck, which was unresponsive to treatment, dysphagia, and deterioration in general condition. Thoracic radiographs showed two soft tissue densities, about $10 \mathrm{~cm}$ in diameter, in the left caudodorsal lung. The goat was euthanized and a necropsy was carried out. The two lesions in the left caudodorsal lung were round, firm and clearly demarcated from the surrounding lung tissue. They contained purulent material and compromised about $70 \%$ of the diaphragmatic lung lobe. Histological examination of the lesions revealed a dense network of hyphae characteristic of Mucorales spp.
\end{abstract}

DOI: https://doi.org/10.1024/0036-7281/a000249

Other titles: Necrotizing granulomatous pneumonia caused by fungal infection in a goat

Posted at the Zurich Open Repository and Archive, University of Zurich

ZORA URL: https://doi.org/10.5167/uzh-50869

Journal Article

Accepted Version

Originally published at:

Wapf, P; Ossent, Pete; Scharf, G; Braun, Ueli (2011). Granulomatös-nekrotisierende Pneumonie durch Schimmelpilzinfektion bei einer Ziege. Schweizer Archiv für Tierheilkunde, 153(10):463-466.

DOI: https://doi.org/10.1024/0036-7281/a000249 
Granulomatös-nekrotisierende Pneumonie durch Schimmelpilzinfektion bei einer Ziege P. Wapf ${ }^{1}$, P. Ossent ${ }^{2 *}$, G. Scharf ${ }^{3}$, U. Braun ${ }^{1}$

${ }^{1}$ Departement für Nutztiere, ${ }^{2}$ Institut für Veterinärpathologie und ${ }^{3}$ Departement für Kleintiere der Universität Zürich

Schlüsselwörter: Granulomatöse Pneumonie, Schimmelpilze, Mucorales, Pneumomykose, Ziege

Necrotizing granulomatous pneumonia caused by fungal infection in a goat

Keywords: Granulomatous pneumonia; Fungus; Mucorales; Pneumomycosis; Goat

* Leider vor dem Erscheinen der Arbeit verstorben. 


\section{$1 \quad$ Einleitung}

2 Pneumonien sind bei Ziegen häufig vorkommende Erkrankungen, wobei die wichtigsten

3 Erreger Parasiten (Protostrongyliden), Bakterien (Pasteurellen, Corynebakterien und

4 Mycoplasmen) und Retroviren (Radostits et al., 2007) sind. Pneumonien durch Pilze

5 sind sehr selten, meist handelt es sich dabei um opportunistische Infektionen (Matthews,

6 2009). Betroffen sind vor allem Patienten mit einem beeinträchtigten Immunsystem

7 sowie Neonaten und Jungtiere (Virally et. al., 2002; Radostits et al., 2007). Da über eine

8 pulmonale Mucormykose bei einer Ziege keine Literatur vorliegt, war es das Ziel dieser

9 Arbeit, den Verlauf und das Ausmass dieser Infektion aufzuzeigen.

10 Anamnese

11 Eine 2.5-jährige weibliche Ziege der Rasse Anglo-Nubier wurde dem Privattierarzt

12 aufgrund von Umfangsvermehrungen am Kopf vorgestellt. Sie stammte aus einem

13 Betrieb mit 38 Milchziegen. Die Tiere wurden in einem Laufstall gehalten, im Sommer

14 geweidet und im Winter regelmässig ins Freie gelassen. Die Fütterung bestand aus Heu

15 und Kraftfutter. Der Rest der Herde war unauffällig. Das Ergebnis der bakteriellen

16 Untersuchung aus der Umfangsvermehrung am Kopf ergab Actinomyces ssp. Die Ziege

17 wurde mit Penicillin/Streptomycin (Pen/Strep 20/20 ${ }^{\circledR}$; Veterinaria AG) behandelt. Trotz

18 dieser Therapie kam es zu einer Verschlechterung des Allgemeinbefindens. Das Tier

19 zeigte zunehmend Mühe beim Schluckakt und wurde zur weiteren Abklärung ans

20 Tierspital Zürich überwiesen.

\section{Klinik und Laboruntersuchung}

22 Das Allgemeinbefinden und die Fresslust waren leicht reduziert. Die rektale

23 Körpertemperatur betrug $38.4{ }^{\circ} \mathrm{C}$. An beiden Unterkiefern und im rechten

24 Kehlkopfbereich waren mehrere, 3- $4 \mathrm{~cm}$ grosse rundliche, nicht schmerzhafte, weiche und verschiebliche Umfangsvermehrungen palpierbar. Die Mandibularlymphknoten

26 waren mittelgradig, die Buglymphknoten leichtgradig vergrössert. Die Atemfrequenz

27 betrug 36 Atemzüge pro Minute und die Ziege hustete spontan. Bei der Auskultation der

28 Lunge wurde verstärktes Vesikuläratmen festgestellt. Dies war auf der linken Seite

29 ausgeprägter als auf der rechten. Das aufgenommene Heu wurde langsam gekaut und

30 mit Mühe geschluckt. Mehrmals konnte Regurgitieren beobachtet werden. Aus den

31 entnommenen Tupferproben der Abszesse im Kehlkopfbereich wurden Staphylococcus 
epidermidis - Keime nachgewiesen, die im Antibiogramm auf sämtliche Antibiotika

33 empfindlich waren.

\section{Röntgenologische und sonographische Untersuchungen}

35 Aufgrund der Befunde am Atemapparat wurde die Lunge in zwei Ebenen geröntgt: In

36 der laterolateralen Aufnahme waren im kaudodorsalen Lungenfeld zwei weichteildichte,

37 gut abgrenzbare, ungefähr $10 \mathrm{~cm}$ grosse Massen sichtbar (Abb. 1). In der dorsoventralen

38 Aufnahme lagen die Massen im Bereich des linken Lungenfeldes und waren jedoch

39 schlecht abgrenzbar. Differentialdiagnostisch wurden die Massen als Abszesse,

40 Granulome, eingeblutete Bullae oder als primäre oder metastatische Neoplasie

41 interpretiert.

42 Sonographisch waren die Umfangsvermehrungen im Unterkieferbereich als in der

43 Subcutis gelegene, abgekapselte Kavernen mit hypoechogenem Inhalt sichtbar, der

44 regelmässig mit hyperechogenen Anteilen durchsetzt war. Bei der sonographischen

45 Untersuchung des Thorax war die Pleura dorsal im linken Interkostalraum ohne

46 Reverberationslinien zu sehen. Ventral hingegen erschien die Lunge wie ein

47 parenchymatöses Organ mit echoarmen Bezirken (Abb. 2). Die subcutanen

48 Umfangsvermehrungen wurden als Abszesse, die Veränderungen im Thoraxbereich als

49 Abszesse, Granulome oder Neoplasien interpretiert. Die Aspiration der Knoten im

50 Kehlkopfbereich ergab eine geruchlose, serös-rötliche Flüssigkeit, die mit festen

51 Krümeln durchsetzt war.

\section{Klinische Diagnose}

53 Aufgrund der Befunde wurde die Verdachtsdiagnose Abszesse in der Kopf- und

54 Halsregion sowie in der Lunge gestellt. Infolge infauster Prognose wurde das Tier

55 euthanasiert und seziert.

\section{Pathologisch-anatomische Untersuchung}

57 Die linke Lungenseite war deutlich vergrössert und zeigte im Anschnitt zwei

58 hintereinander gelegene, derbe, beige, kugelige Massen von ca. $10 \mathrm{~cm}$ Durchmesser, die

59 im Zentrum teilweise eitrig eingeschmolzen waren (Abb. 3). Die Massen setzten sich

60 deutlich vom Lungengewebe ab und verdrängten mehr als $70 \%$ des linken

61 Zwerchfelllappens. Der kaudale Knoten setzte sich ins Mediastinum fort, wo er mit dem

62 ebenfalls stark vergrösserten Mediastinallymphknoten verbunden war. Dieser umschloss 
63 den Ösophagus und war stellenweise mit dem Perikard verwachsen. Der Ösophagus war

64 um ein Drittel verengt. Zusätzlich befanden sich im Myokard zwei weissliche, derbe,

65 kugelige Gebilde von $2 \mathrm{~cm}$ Durchmesser. Die Knoten in Lunge, Lymphknoten und

66 Myokard wurden mikroskopisch als Granulome identifiziert. Sie wiesen eine

67 grossflächige zentrale Nekrose, massenhaft Fremdkörperriesenzellen und eine dichte

68 Besiedlung mit Pilzhyphen auf (Abb. 4). Anhand der morphologischen Merkmale der

69 Hyphen liessen sich die Pilze der Ordnung Mucorales zuordnen.

70 Aufgrund sämtlicher Befunde wurde die Diagnose einer hochgradigen, granulomatösen,

71 nekrotisierenden, mykotischen Pneumonie gestellt. Zudem eine Lymphadenitis und

72 Myocarditis (Mucormykose) sowie bilaterale Abszesse der Unterhaut im Unterkiefer-

73 und Kehlkopfbereich (verursacht durch Staphylococcus epidermidis).

\section{Diskussion}

75 Pilzinfektionen der Lunge wurden meist als Sekundärinfektion einer bestehenden

76 Erkrankung oder nach Therapie einer solchen beschrieben (Spira et al., 2002; Virally et

77 al., 2002; Radostits et al., 2007; Smith und Sherman, 2009; Woolums und Wikse, 2009).

78 Insbesondere der Einsatz von Antibiotika, Zytostatika, aber auch lang andauernde

79 Corticosteroidapplikationen sind als begünstigende Faktoren für Pilzinfektionen (Spira

80 et al., 2002; Virally et al., 2002; Al-Ajam et al., 2006) bekannt. In der Humanmedizin

81 sind Patienten mit immunschwächenden Erkrankungen, Organtransplantierte und

82 insbesondere Patienten mit Diabetes mellitus besonders empfänglich für

83 Mucorpneumonien (Virally et al., 2002; Di Carlo et al., 2010). Pilze der Ordnung

84 Mucorales sind aerober und opportunistischer Natur und finden in den Lungen ein sehr

85 passendes Umfeld (Franquet et al., 2004). Bei Wiederkäuern können Futtermittel und

86 Einstreu als Nährboden der Pilze und somit als Infektionsquellen angesehen werden

87 (Radostits et al., 2007). Es wird auch berichtet, dass die Pilze im Panseninhalt gesunder

88 Wiederkäuer vorkommen und bei Erkrankungen zu Sekundärinfektionen führen können

89 (Pohlenz et al., 1973; Smith und Sherman, 2009). Die Schimmelpilze können durch

90 Inhalation von Panseninhalt, beispielsweise durch Verschlucken in die Lunge gelangen.

91 Empfindlich sind vor allem Ziegen, die mit Antibiotika und Corticosteroiden behandelt

92 werden (Smith und Sherman, 2009). Erkrankungen die das Immunsystem beeinflussen

93 und oder Erkrankungen die immunsuppressive Therapien mit sich ziehen, spielen bei

94 Ziegen eine untergeordnete Rolle. Diabetes mellitus ist selten, wird aber bei Ziegen 
95 beschrieben (Braun et al., 2008), bei diesem Tier wurden keine Anzeichen eines

96 Diabetes mellitus festgestellt. Die bakteriell bedingte Infektion am Kopf und deren

97 Therapie mit Antibiotika wären als mögliche Ursachen für die Entstehung der

98 Pneumomykose annehmbar. Eine saisonal bedingte Häufung von Pilzinfektionen der

99 Atemwege werden in der Humanmedizin zusätzlich zu den durch Krankheiten

100 prädisponierten Patienten beobachtet (Al-Ajam et al., 2006). Bei dieser Ziege könnten die

101 jahreszeitlich bedingte Umstellung von Weidehaltung auf Stallhaltung und die

102 Heufütterung als weitere, die Pilzinfektion begünstigende Faktoren in Betracht gezogen

103 werden.

104 Infektionen mit Pilzen führen zu einem uneinheitlichen Krankheitsbild, lassen

105 bestenfalls eine ätiologische Verdachtsdiagnose zu (Müller et al., 2001; Ainsworth und

106 Hackett, 2004). In der Veterinärmdizin werden sie meist erst durch Untersuchungen

107 post mortem diagnostiziert (Blanco und Garcia, 2011). In diesem Fall führten die den

108 Ösophagus umschliessenden Massen zu den beobachteten Schluckbeschwerden und

109 zum Regurgitieren. Röntgenologisch kann sich eine Pneumomykose als Granulom

110 darstellen (Franquet et al., 2004), sie kann aber auch zu flächenhaften Veränderungen

111 führen (Virally et al., 2002). Die definitive Diagnose kann nur durch Biopsie respektive

112 Kultur oder histopathologische Untersuchung gestellt werden (Müller et al., 2001; Spira

113 et al., 2002). Mucormykosen stellen sich dabei als charakteristische Hyphen dar

114 (Chihaya et al., 1992; Müller et al., 2001). Die Therapie der Wahl besteht beim

115 Menschen in einer hochdosierten systemischen Gabe von Amphotericin B und einem

116 chirurgischen Debridement (Donado-Una et al., 2002). Beim Grosstier ist lediglich beim

117 Pferd die Anwendung von Amphotericin B beschrieben (Radostits et al., 2007). Die

118 Behandlung ist jedoch sehr teuer und die Prognose ist zweifelhaft (Ainsworth und

119 Hackett, 2004).

\section{Literaturverzeichnis}

121 Ainsworth, D. M., Hackett, R. P.: Disorders of the respiratory system. In: Equine

122 Internal Medicine. Eds. S. M. Reed, W. M. Bayly \& D.C. Sellon, Saunders, St. Louis, $1232004,289-355$.

124 Al-Ajam, M. R., Bizri, A. R., Mokhbat, J. Weedon, J., Lutwick, L.: Mucormycosis in the 125 Eastern Mediterranean: a seasonal disease. Epidemiol. Infect., 2006, 134: 341- 346. 
126 Blanco, J. L., Garcia, M. E.: Are fungi important in veterinary medicine? Vet. J., 2011, 127 187, 10- 11.

128 Braun U., Gansohr B., Seidel M., Dumelin J., Wenger B., Schade B., Pospischil A.:

129 Diabetes mellitus Typ 1 bei einer Ziege. Schweiz. Arch. Tierheilk., 2008, 150: 608-612.

130 Chihaya, Y., Okada, H., Matsukawa, K., Matsui, Y.: Disseminated mycosis in cattle. A

131 study on nine autopsy cases. J. Vet. Med. Sci., 1992, 53: 1051-1058.

132 Di Carlo, P., Cabibi, D., La Rocca A. M., De Luca, D., La Licata, F., Sacco, E.: Post-

133 bronchoscopy fatal hemorrhage in a woman with bronchopulmonary mucormycosis: a

134 case report. J. Med. Case Report, 2010, 4: 398

135 Donado-Una, J. R., Diaz-Hellin, V., Lopez-Encuentra, A., Echave-Sustaeta, J.:

136 Persistent cavitations in pulmonary mucormycosis after apparently successful

137 amphotericin B. Eur. J. Cardiothoracic Surg., 2002, 21: 940-942.

138 Franquet, T., Gimenez, A., Hidalgo, A.: Imaging of opportunistic fungal infections in 139 immunocompromised patients. Eur. J. Radiol., 2004, 51: 130-138.

140 Matthews, J. G.: Diseases of the Goat. Blackwell Science Ltd., Oxford, 2009, 298-311.

141 Müller, M., Nuss, K., Fuhrmann, U., Herrmanns, W.: Systemic zygomatosis as a result 142 of left displacement of the abomasum in a cow. Tierärztl. Praxis, 2001, 29 (G): 62-67.

143 Pohlenz, J., Ehrensperger, F., Breer, C.: Sudden death due to mucormycosis of the 144 omasum in cattle. Schweiz. Arch. Tierheilk., 1973, 115: 161-168.

145 Radostits, O. M., Blood, D. C., Gay, C. C.: Fungal diseases. In: Veterinary Medicine. A 146 textbook of the diseases of cattle, horses, sheep, pigs, and goats. Eds: O. M. Radostits, 147 D. C. Blood, C. C. Gay, W. B. Saunders, London, 2007, 1471-1481.

148 Smith, M. C., Sherman, D. M.: Lower respiratory tract diseases. In: Goat Medicine. 149 Wiley-Blackwell, Iowa, 2009, 348.

150 Spira, S., Brecher, S., Karlinsky, J.: Pulmonary mucormycosis in the setting of chronic 151 obstructive pulmonary disease. Respiration, 2002, 69: 560-563.

152 Virally, M., Rivelline, J., Virally, J., Chevojon, P., Regnard, J., Belmekki, A., Devidas, 153 A.: Pulmonary mucormycosis in a diabetic patient with HIV. Diabetes Care, 2002, 25: 1542105. 
155 Woolums, A., Wikse, S.: Mycotic pneumonias. In: Large Animal Internal Medicine. Ed.

156 B. P. Smith., Mosby Elsevier, St. Louis, 2009, 659.

157 Korrespondenz

158 Pascale Wapf

159 Departement für Nutztiere der Vetsuisse Fakultät Zürich

160 Winterthurerstrasse 260

1618057 Zürich

162 E-Mail: pascale.wapf@gmx.ch 


\section{Abstract}

This report describes a 2.5-year-old goat with a granulamatous necrotising pneumonia because of an infection with fungus. The goat was referred to our clinic because of swellings on the head and neck. In spite of treating, the general condition deteriorated and swallowing became difficult. The clinical findings showed these swellings, coughing and increased breath sounds. The goat swallowed with great difficulty and regurgitated. The radiographs showed two soft tissue densities that were $10 \mathrm{~cm}$ in diameter in the caudodorsal part of the left lung. Based on the clinical findings, a tentative diagnosis abscesses of the head- neck and lungs was made and euthanasia and postmortem examination were carried out. The left lung showed two spherical masses with $10 \mathrm{~cm}$ in diameters and occupying $70 \%$ of the cent lobe. Histological examination of the masses revealed a dense population of fungal hyphae of the order

Mucorales.

\section{Zusammenfassung}

In der vorliegenden Arbeit wird ein Fall einer granulomatös- nekrotisierenden Pneumonie bei einer 2.5 Jahre alten Ziege durch eine Infektion mit Schimmelpilzen beschrieben. Die Ziege wurde wegen Umfangsvermehrungen am Kopf und am Hals, die trotz Behandlung zu einer Verschlechterung des Allgemeinzustandes und Schluckbeschwerden geführt hatten, in die Klinik überwiesen. Bei der klinischen Untersuchung fielen neben den Umfangsvermehrungen Husten sowie ein verstärktes Vesikuläratmen auf Die Ziege konnte nur mit Mühe schlucken und regurgitierte. Die röntgenologische Untersuchung zeigte im kaudodorsalen, linken Lungenfeld zwei $10 \mathrm{~cm}$ grosse weichteildichte Massen. Aufgrund der klinischen Befunde wurde die Diagnose Abszesse in Kopf- und Halsregion sowie in der Lunge gestellt. Die Ziege wurde euthanasiert und pathologisch-anatomisch untersucht. Im Bereich der linken Lungenseite befanden sich zwei derbe kugelige Massen mit derbem, eitrig eingeschmolzenem Inhalt von $10 \mathrm{~cm}$ Durchmesser, die sich deutlich vom Lungengewebe absetzten und $70 \%$ des Zwerchfelllappens verdrängten. Die histologische Untersuchung ergab eine dichte Besiedelung der Massen mit Pilzhyphen der Ordnung Mucorales. 


\section{Zusammenfassung}

In der vorliegenden Arbeit wird ein Fall einer granulomatös- nekrotisierenden Pneumonie bei einer 2.5 Jahre alten Ziege durch eine Infektion mit Schimmelpilzen beschrieben. Die Ziege wurde wegen Umfangsvermehrungen am Kopf und am Hals, die trotz Behandlung zu einer Verschlechterung des Allgemeinzustandes und Schluckbeschwerden geführt hatten, in die Klinik überwiesen. Bei der klinischen Untersuchung fielen neben den Umfangsvermehrungen Husten sowie ein verstärktes Vesikuläratmen auf. Die Ziege konnte nur mit Mühe schlucken und regurgitierte. Die röntgenologische Untersuchung zeigte im kaudodorsalen, linken Lungenfeld zwei $10 \mathrm{~cm}$ grosse weichteildichte Massen. Aufgrund der klinischen Befunde wurde die Diagnose Abszesse in Kopf- und Halsregion sowie in der Lunge gestellt. Die Ziege wurde euthanasiert und pathologisch-anatomisch untersucht. Im Bereich der linken Lungenseite befanden sich zwei derbe kugelige Massen mit derbem, eitrig eingeschmolzenem Inhalt von $10 \mathrm{~cm}$ Durchmesser, die sich deutlich vom Lungengewebe absetzten und $70 \%$ des Zwerchfelllappens verdrängten. Die histologische Untersuchung ergab eine dichte Besiedelung der Massen mit Pilzhyphen der Ordnung Mucorales.

\section{Summary}

This case report describes the clinical and postmortem findings in a 2.5-year-old goat with necrotizing granulomatous pneumonia caused by fungal infection. The goat was referred to our clinic because of swelling of the head and neck, which was unresponsive to treatment, dysphagia, and deterioration in general condition. Clinical examination also revealed coughing, increased breath sounds and marked difficulty in swallowing and regurgitation. Thoracic radiographs showed two soft tissue densities, about $10 \mathrm{~cm}$ in diameter, in the left caudodorsal lung. A tentative diagnosis of abscess formation in the head and neck region and left lung was made. The goat was euthanased and a necropsy was carried out. The two lesions in the left caudodorsal lung were round, firm and clearly demarcated from the surrounding lung tissue. They contained purulent material and compromised about $70 \%$ of the diaphragmatic lung lobe. Histological examination of the lesions revealed a dense network of hyphae characteristic of Mucorales spp. 
$\downarrow$

$y$ 


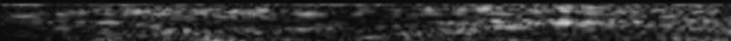

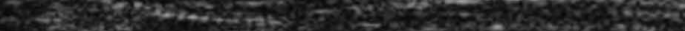

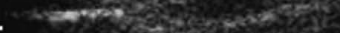

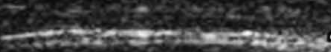

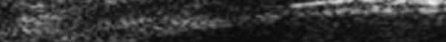
$2-$

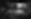
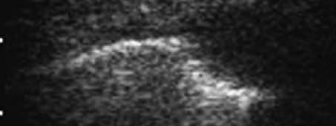

\section{$x$}

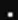

•

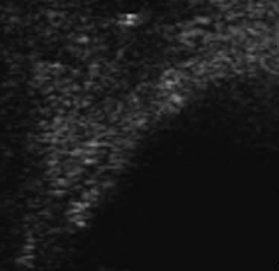

•

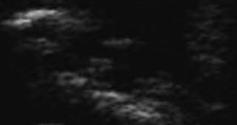

$-2$

-

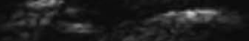

4

- 
wheratsons 
